\title{
Preparation and Decay of a Single Quantum of Vibration at Ambient Conditions
}

\author{
Santiago Tarrago Velez, ${ }^{1}$ Kilian Seibold, ${ }^{1}$ Nils Kipfer, ${ }^{1}$ Mitchell D. Anderson, ${ }^{1}$ \\ Vivishek Sudhir, ${ }^{2,3}$ and Christophe Galland $\oplus^{1}$ \\ ${ }^{1}$ Institute of Physics, Ecole Polytechnique Fédérale de Lausanne (EPFL), CH-1015 Lausanne, Switzerland \\ ${ }^{2}$ LIGO Laboratory, Massachusetts Institute of Technology, Cambridge, Massachusetts 02139, USA \\ ${ }^{3}$ Department of Mechanical Engineering, Massachusetts Institute of Technology, \\ Cambridge, Massachusetts 02139, USA
}

(Received 7 November 2018; revised manuscript received 29 May 2019; published 7 October 2019)

A single quantum of excitation of a mechanical oscillator is a textbook example of the principles of quantum physics. But mechanical oscillators, despite their pervasive presence in nature and modern technology, do not generically exist in an excited Fock state. In the past few years, careful isolation of gigahertz-frequency nanoscale oscillators has allowed experimenters to prepare such states at millikelvin temperatures. These developments illustrate the tension between the basic predictions of quantum mechanics-which should apply to all mechanical oscillators even at ambient conditions-and the extreme conditions required to observe those predictions. We resolve the tension by creating a single Fock state of a $40-\mathrm{THz}$ vibrational mode in a crystal at room temperature and atmospheric pressure. After exciting a bulk diamond with a femtosecond laser pulse and detecting a Stokes-shifted photon, the Ramanactive vibrational mode is prepared in the Fock state $|1\rangle$ with $98.5 \%$ probability. The vibrational state is then mapped onto the anti-Stokes sideband of a subsequent pulse, which when subjected to a Hanbury BrownTwiss intensity correlation measurement reveals the sub-Poisson number statistics of the vibrational mode. By controlling the delay between the two pulses, we are able to witness the decay of the vibrational Fock state over its 3.9-ps lifetime at ambient conditions. Our technique is agnostic to specific selection rules, and should thus be applicable to any Raman-active medium, opening a new general approach to the experimental study of quantum effects related to vibrational degrees of freedom in molecules and solidstate systems.

DOI: 10.1103/PhysRevX.9.041007

\section{INTRODUCTION}

The observation of inelastic scattering of photons from ensembles of atomic-scale particles was an early triumph of quantum theory. Within a few years, experiments by Compton [1] and Raman and Krishnan [2] showed that photons can exchange energy and momentum with material particles in the manner described by quantum mechanics. At optical frequencies, Raman scattering, the dominant effect, is an expression of the universal idea that a mechanical vibration phase modulates the outgoing light, resulting in two scattered sidebands [Figs. 1(a) and 1(b)]. In a quantum description, the upper ("anti-Stokes") sideband arises from the annihilation of a quantum of vibration, while the lower ("Stokes") arises from its creation [Fig. 1(c)].

Published by the American Physical Society under the terms of the Creative Commons Attribution 4.0 International license. Further distribution of this work must maintain attribution to the author(s) and the published article's title, journal citation, and DOI.
Subject Areas: Condensed Matter Physics, Photonics, Quantum Physics

Leveraging the Raman interaction, a variety of pumpprobe measurements have been implemented to study vibrational dynamics in crystals and molecules. For example, incoherent phonons generated by the decay of electron-hole pairs can be probed by time-resolved anti-Stokes scattering [3-6]. Several techniques have also been developed to study coherent states of the vibrational modes. The most popular is time-resolved coherent anti-Stokes Raman scattering, where a large coherent phonon population is excited by a pair of laser pulses and is probed by a delayed pulse [7]. Another technique-transient coherent ultrafast phonon spectroscopy-uses the interference of the Stokes photons from the spontaneous Raman scattering of two coherent pumps to determine the decoherence of the vibrational mode [8,9]. While these techniques reveal the timescales over which the vibration decays or loses its phase coherence, observing single quanta of the vibration itself has proved far more elusive.

To illustrate the difficulty, consider that on the one hand, internal vibrational modes of crystals and molecules with oscillation frequencies in the 10-100 THz range ubiquitously 
(a)

(b)
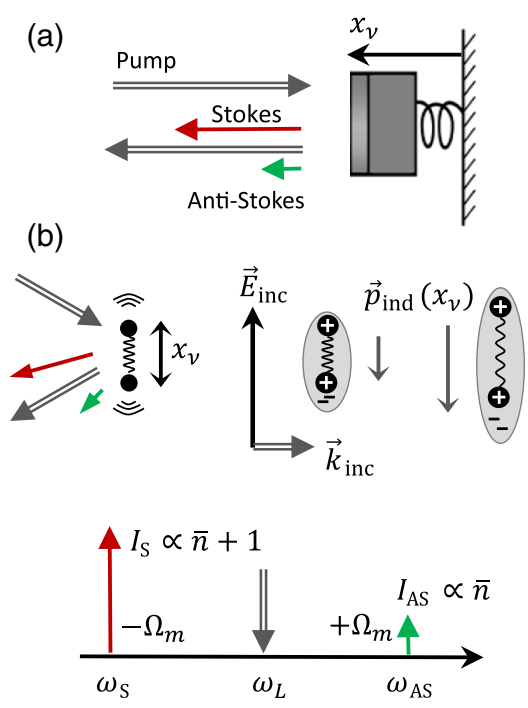

(c)

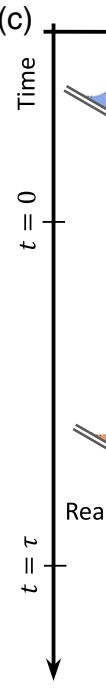

Space

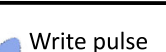

Write pulse

Write puls

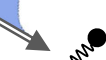

Stokes $\left(\hat{a}_{\mathrm{S}}\right)$

«...M/

$\because$

Vibration

(b) $\because \cdots$<smiles>[C]1CCC1</smiles>

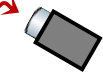

Herald detector

Success
|0
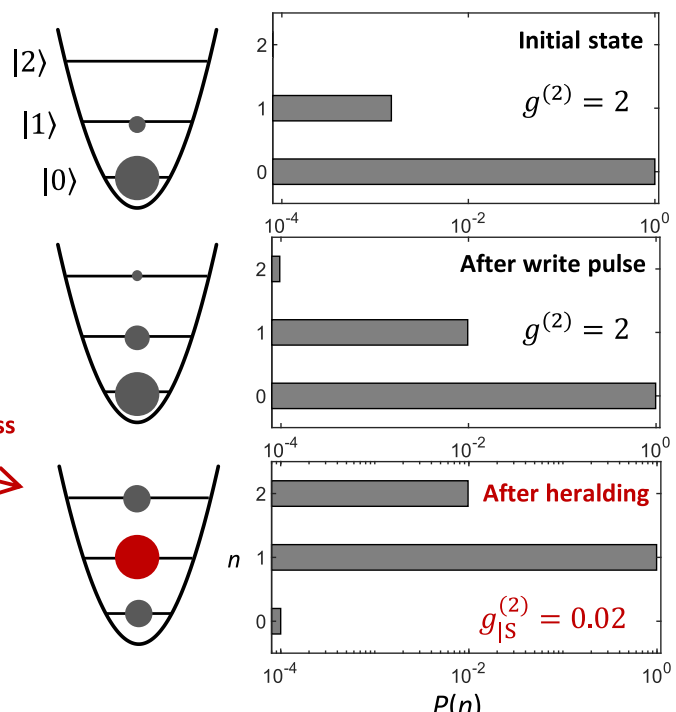

FIG. 1. Concept of the experiment. (a) When monochromatic light reflects from an oscillating mirror, it acquires two Raman sidebands due to phase modulation (to first order in interaction strength). (b) When light interacts with a polarizable oscillator, whose induced polarization $\vec{p}_{\text {ind }}$ depends on its internal coordinate $x_{\nu}$, it undergoes a phase modulation at the oscillator frequency, leading to the appearance of Stokes and anti-Stokes Raman sidebands in the scattered light. The anti-Stokes/Stokes intensity ratio is proportional to $\bar{n} /(\bar{n}+1)$, where $\bar{n}$ is the mean excitation number (or occupancy) of the vibrational mode. (c) Time evolution of a single repetition of the experiment showing the interaction of the write pulse, the subsequent detection of Stokes photons for heralding, and the final read pulse followed by measurement of two-photon correlation in the emitted anti-Stokes light. (d) Evolution of the probability $P(n)$ of finding the vibrational mode in the $n$th energy eigenstate during the different steps. The system is initially in a thermal state with $\bar{n}=1.5 \times 10^{-3}$ (for a mode frequency of $40 \mathrm{THz}$ at $295 \mathrm{~K})$. After the write pulse, the marginal state of the vibration is also thermal with $\bar{n}=[p /(1-p)]=$ $10^{-2}$ in this example (here $p$ is the interaction probability). Finally, after heralding, the distribution becomes peaked at $n=1$. The residual vacuum component is due to detection noise. For each distribution, we give the corresponding value of the vibrational mode's intensity correlation function $g^{(2)}$.

and naturally exist in their quantum ground state at room temperature. But unless they are individually addressed and resolved within their coherence time, the ensemble average over unresolved vibrational modes precludes the observation of individual quanta. Despite this challenge, a Raman-active vibration featuring a specific form of internal nonlinearity was prepared in a squeezed state by optical excitation [10], while polarization-selective Raman interactions were used to observe two-photon interferences mediated by a vibrational mode [11]. On the other hand, nanofabricated mechanical oscillators can be susceptible to a universal radiation pressure interaction with light, especially with the intense fields stored in an optical cavity [12]. However, their relatively low frequency (megahertz to gigahertz) means that thermal energy at room temperature is larger than the energy of a single vibrational quantum, making quantum state manipulation difficult or impossible under ambient conditions. Precise measurements of mechanical motion at room temperature have recently revealed quantum characteristics of the underlying radiation pressure interaction [13-15]. But it is only through deep cryogenic operation that quantum states of motion of nanoscale oscillators have been prepared in recent years [16-20]. Thus, the quest to prepare quantum states of commonly available mechanical oscillators at ambient conditions remains largely open.
Here we prepare a nonclassical state of an internal vibration of a diamond crystal at room temperature. In a scheme inspired by the Duan-Lukin-Cirac-Zoller protocol [21,22], a femtosecond laser pulse (the "write" pulse hereafter) first creates an excitation from the ambient motional ground state with a probability $p \ll 1$ [see Fig. 1(c)]. Detection of the emitted Stokes photon heralds the success of this step, while the choice of the spectral window for detection fixes the specific vibrational mode of the system under study. To verify that only a single quantum of vibration was excited, a second laser pulse (the "read" pulse) retrieves it as an anti-Stokes photon. The probability of having two or more anti-Stokes photons, and therefore two or more quanta of vibrations, is obtained by performing a Hanbury BrownTwiss (HBT) intensity correlation measurement on the antiStokes photons conditioned on the heralding signal $[23,24]$. We observe sub-Poissonian statistics of the heralded vibrational state, a result consistent with having prepared the Fock state $|1\rangle$ of the vibrational mode (the first excited energy eigenstate). Finally, by changing the delay between the write and read pulses, we probe the decay of the single vibrational quantum, with $\approx 200$ fs resolution.

In contrast to previous quantum optics experiments on Raman-active vibrational modes that were restricted to molecular or crystal structures exhibiting particular 
polarization rules $[11,25-30]$ or vibrational nonlinearities [10], our technique is agnostic to these details, and can be employed on any Raman-active subject. It opens a plethora of opportunities to study vibrational quantum states and dynamics in other crystals and in molecules, and can readily be extended to create vibrational twomode entangled states [31] and test the violation of Bell inequalities $[32,33]$ at room temperature in a number of widely available systems.

\section{THEORETICAL DESCRIPTION}

The Raman interaction between a single vibrational mode and an optical field leads to the creation of an anti-Stokes (Stokes) photon commensurate with the destruction (creation) of a vibrational quantum. In our experiment the Raman interaction is driven by an optical field that may be either a "write" (superscript $w$ ) or a "read" (superscript $r$ ) pulse defined by the spatial and temporal mode of a mode-locked laser whose beam is focused onto the sample. These two incident fields are described by the annihilation operators $\hat{a}^{w, r}$. The interaction leads to the generation of Stokes and anti-Stokes photons whose spatial mode is postselected by projecting the focal spot onto the core of a single-mode optical fiber. The Stokes (antiStokes) fields are modeled by annihilation operators $\hat{a}_{\mathrm{S}}^{w, r}$ $\left(\hat{a}_{\mathrm{AS}}^{w, r}\right)$. Because of conservation of energy and momentum in the Raman scattering process, the detection of these scattered fields as described above defines a single spatiotemporal mode of the vibration that is the subject of the experiment, and which we describe by its annihilation operator $\hat{b}$. The Raman interaction is modeled by the Hamiltonian [34]

$$
\begin{aligned}
& \hat{H}_{\mathrm{int}}^{w}=i \hbar\left[G_{\mathrm{S}}^{w} \hat{a}^{w} \hat{b}^{\dagger}\left(\hat{a}_{\mathrm{S}}^{w}\right)^{\dagger}+G_{\mathrm{AS}}^{w} \hat{a}^{w} \hat{b}\left(\hat{a}_{\mathrm{AS}}^{w}\right)^{\dagger}\right]+\text { H.c., } \\
& \hat{H}_{\mathrm{int}}^{r}=i \hbar\left[G_{\mathrm{S}}^{r} \hat{a}^{r} \hat{b}^{\dagger}\left(\hat{a}_{\mathrm{S}}^{r}\right)^{\dagger}+G_{\mathrm{AS}}^{r} \hat{a}^{r} \hat{b}\left(\hat{a}_{\mathrm{AS}}^{r}\right)^{\dagger}\right]+\text { H.c., }
\end{aligned}
$$

where the coupling rates $G_{\mathrm{S}}^{w, r}$ and $G_{\mathrm{AS}}^{w, r}$ relate to the Raman activity of the vibrational mode.

None of the four processes described by Eq. (1) is resonant since we work at photon energies well below the band gap of diamond $(5.47 \mathrm{eV})$. However, because we spectrally filter and detect only the two modes $a_{\mathrm{S}}^{w}$ and $a_{\mathrm{AS}}^{r}$, all essential results of our measurements can be described by the Hamiltonian

$$
\begin{aligned}
& \hat{H}_{\mathrm{int}}^{w}=i \hbar g_{\mathrm{S}}^{w} \hat{b}^{\dagger} \hat{a}_{\mathrm{S}}^{\dagger}+\text { H.c., } \\
& \hat{H}_{\mathrm{int}}^{r}=i \hbar g_{\mathrm{AS}}^{r} \hat{b} \hat{a}_{\mathrm{AS}}^{\dagger}+\text { H.c., }
\end{aligned}
$$

where we defined the effective coupling rate, $g_{\mathrm{S}, \mathrm{AS}}^{w, r} \equiv$ $G_{\mathrm{S}, \mathrm{AS}}^{w, r} \sqrt{n_{p}^{w, r}}$, corresponding to a classical excitation for the write or read pulses with $n_{p}^{w, r}$ photons per pulses, and used the shorter notation $\hat{a}_{\mathrm{S}} \equiv \hat{a}_{\mathrm{S}}^{w}, \hat{a}_{\mathrm{AS}} \equiv \hat{a}_{\mathrm{AS}}^{r}$. In fact, this scenario is equivalent to the linearized radiation-pressure interaction [12], or Raman processes in atomic ensembles [21], where an optical cavity (in the former instance) or electronic resonance (in the latter) suppresses nonresonant terms.

Note that the Hamiltonian in Eq. (2) neglects higher-order interactions, in particular, the creation of correlated Stokesanti-Stokes pairs during a single pulse (write or read) via phonon-assisted four-wave mixing [35-40]. In our experiment, the photon flux due to this higher-order interaction constitutes an effective background noise [41]. Other extraneous sources of photons, such as from residual $\chi^{(3)}$ nonlinearities, or fluorescence, also lead to an excess background noise. The spontaneous Stokes signal scales linearly with laser power since it is proportional to $n_{p}^{w}(\bar{n}+1) \approx n_{p}^{w}$, where $\bar{n} \ll 1$ is the average occupation of the vibrational mode. In our experiment, the spontaneous Raman signal is much stronger than any of the parasitic processes described above, so that the fidelity of the heralded state is only marginally affected by noise. However, the anti-Stokes signal, being proportional to $n_{p}^{r} \bar{n}$, is significantly weaker, so that noise cannot be neglected in this case. The measured normalized probability of detecting two anti-Stokes photons should therefore be considered an upper bound for the corresponding probability of having two vibrational quanta. In the calculations presented below, the noise terms are accounted for in order to faithfully describe the experiment.

\section{A. Write operation}

The first step in an iteration of the experiment is the excitation of the vibrational mode by a write pulse. The resulting dynamics of the vibrational and Stokes modes is governed by $H_{\text {int }}^{w}=i \hbar g_{\mathrm{S}}^{w} \hat{b}^{\dagger} \hat{a}_{\mathrm{S}}^{\dagger}+$ H.c., which has the form of a two-mode squeezing interaction, and leads to the creation of maximally correlated pairs of vibrational and Stokes excitations. When both modes are initially in the vacuum state $|\mathrm{vac}\rangle$, the final state after the write pulse is [24]

$$
|\Psi\rangle_{\mathrm{S}, b}=\sqrt{1-p} \sum_{n=0}^{\infty} \sqrt{p^{n}}|n, n\rangle_{\mathrm{S}, b},
$$

where $|n, n\rangle_{\mathrm{S}, b} \equiv\left[\left(\hat{b}^{\dagger} \hat{a}_{\mathrm{S}}^{\dagger}\right)^{n} / n !\right] \mid$ vac $\rangle$. For the simple situation of a constant interaction switched on for a duration $T_{w}$, the probability of exciting the state $|1,1\rangle$ is given by, $p=\tanh ^{2}\left(g_{\mathrm{S}}^{w} T_{w}\right)$. For realistic laser pulse shapes, in the linear regime, $T_{w}$ is the effective interaction time defined by the equivalent square pulse that carries the same pulse energy. Ideally, when at least one Stokes photon is detected, the (conditional) state of the vibrational mode becomes (see Supplemental Material, Sec. I [42]) $\rho_{b \mid \mathrm{S}} \approx|1\rangle\langle 1|+p| 2\rangle\langle 2|$, in the limit where $p \ll 1$; crucially, the vacuum component in $|\Psi\rangle_{\mathrm{S}, b}$ has been eliminated based on the presence of a Stokes photon. Dark noise in real photodetectors (modeled as a probability $\pi_{0}$ per pulse) prevents unambiguous discrimination of the vacuum contribution. However, when the total 
Stokes signal is larger than the dark noise, it can be shown (see Supplemental Material, Sec. I.A [42]) that the resulting conditional state,

$$
\hat{\rho}_{b \mid \mathrm{S}} \approx \frac{\pi_{0}}{2 \eta p}|0\rangle\langle 0|+| 1\rangle\langle 1|+p| 2\rangle\langle 2|,
$$

is dominated by the contribution from the pure Fock state $|1\rangle$. Here, $\eta$ is the detection efficiency of the Stokes field, and $\eta p$ is the Stokes detection probability. The signal-to-noise ratio in the Stokes photodetector $\eta p / \pi_{0}$ is larger than $10^{4}$ in our experiment.

\section{B. Read operation}

Once the Stokes photon is detected, a second pulse - the read pulse-is used to retrieve the (conditional) state of the vibrational mode. The dynamics of the anti-Stokes field induced by the read pulse is described by the Hamiltonian $H_{\mathrm{int}}^{r}=i \hbar g_{\mathrm{AS}}^{r} \hat{b} \hat{a}_{\mathrm{AS}}^{\dagger}+$ H.c., which represents a beam splitter interaction between the anti-Stokes and vibrational modes. For an effective interaction time $T_{r}$, the state of the emitted anti-Stokes field is governed by the input-output relation,

$$
\hat{a}_{\mathrm{AS}, \text { out }}\left(T_{r}\right)=\cos (\theta) \hat{a}_{\mathrm{AS}, \text { in }}+\sin (\theta) \hat{b},
$$

where $\theta \equiv g_{\mathrm{AS}}^{r} T_{r}$. The mode $\hat{a}_{\mathrm{AS} \text {,in }}$ describes the input anti-Stokes mode (before the read pulse), which is in the vacuum state.

The crucial aspect of the read operation is that the emitted anti-Stokes field faithfully reflects the number statistics of the vibrational mode. In fact, from Eq. (5) we find that $\left\langle:\left(\hat{a}_{\mathrm{AS}}^{\dagger} \hat{a}_{\mathrm{AS}}\right)^{n}:\right\rangle=\sin ^{2 n}(\theta)\left\langle:\left(\hat{b}^{\dagger} \hat{b}\right)^{n}:\right\rangle$, for any integer $n \geq 1$ (here $::$ denotes normal ordering). This relation expresses the fact that photon counting is insensitive to vacuum noise (in stark contrast to linear detection of the field [43]), so that normalized moments of the photon number of the anti-Stokes field faithfully represent the statistics of the vibrational mode excitation number.

Note that although the interaction between the optical pulses and the vibrational mode is linear [Eq. (2)], the nonlinearity provided by the single-photon detection following the write and read pulses renders the full measurement process effectively nonlinear [44]. It is this single-photon nonlinearity that lies at the conceptual heart of our protocol.

\section{Statistics of the heralded intensity correlation}

Performing the two operations presented above enables the preparation and unambiguous characterization of a vibrational Fock state. Consider the joint state $|\Psi\rangle_{\mathrm{S}, b, \mathrm{AS}}$ for the Stokes field, the vibration, and the anti-Stokes field. A heralded coincidence event occurs when a Stokes photon is detected at time $t=0$, followed, after a time $t$, by coincident detection of a pair of anti-Stokes photons. This heralded coincidence event is represented by the measurement map,

$$
|\Psi\rangle_{\mathrm{S}, b, \mathrm{AS}} \mapsto \hat{d}_{2}(t) \hat{d}_{1}(t) \hat{a}_{\mathrm{S}}(0)|\Psi\rangle_{\mathrm{S}, b, \mathrm{AS}},
$$

where $\hat{d}_{1,2}$ are the operators denoting the anti-Stokes field at the two outputs of a 50/50 beam splitter [see Fig. 1(c)]. The probability of this triple coincidence defines the conditional intensity correlation, and is thus proportional to $\left\langle\hat{a}_{\mathrm{S}}^{\dagger}(0) \hat{d}_{1}^{\dagger}(t) \hat{d}_{2}^{\dagger}(t) \hat{d}_{2}(t) \hat{d}_{1}(t) \hat{a}_{\mathrm{S}}(0)\right\rangle$; here we have used the linearity of quantum mechanics to extend the definition to mixed states as well. Suitably normalizing the expression [45] allows us to define the conditional intensity correlation,

$$
g_{\mathrm{AS} \mid \mathrm{S}}^{(2)}(t) \equiv \frac{\left\langle\hat{a}_{\mathrm{S}}^{\dagger}(0) \hat{d}_{1}^{\dagger}(t) \hat{d}_{2}^{\dagger}(t) \hat{d}_{2}(t) \hat{d}_{1}(t) \hat{a}_{\mathrm{S}}(0)\right\rangle}{\prod_{i}\left[\left\langle\hat{a}_{\mathrm{S}}^{\dagger}(0) \hat{d}_{i}^{\dagger}(t) \hat{d}_{i}(t) a_{\mathrm{S}}(0)\right\rangle\left\langle\hat{a}_{\mathrm{S}}^{\dagger}(0) \hat{a}_{\mathrm{S}}(0)\right\rangle^{-1 / 2}\right]} .
$$

The fields $\hat{d}_{1,2}$ whose intensity cross-correlation is measured can be expressed in terms of the anti-Stokes field $\hat{a}_{\mathrm{AS}}$, which in turn can be expressed in terms of the vibration [via Eq. (5)]; since intensity correlations do not respond to the vacuum, the open port of the beam splitter used in the intensity correlation measurement plays no role, and the conditional correlation above can be written as

$g_{\mathrm{AS} \mid \mathrm{S}}^{(2)}(t)=\frac{\left\langle\hat{a}_{\mathrm{S}}^{\dagger}(0) \hat{b}^{\dagger}(t) \hat{b}^{\dagger}(t) \hat{b}(t) \hat{b}(t) \hat{a}_{\mathrm{S}}(0)\right\rangle}{\left\langle\hat{a}_{\mathrm{S}}^{\dagger}(0) \hat{a}_{\mathrm{S}}(0)\right\rangle^{-1}\left\langle\hat{a}_{\mathrm{S}}^{\dagger}(0) \hat{b}^{\dagger}(t) \hat{b}(t) \hat{a}_{\mathrm{S}}(0)\right\rangle^{2}}$.

After the detection of a Stokes photon (i.e., for $t>0$ ), the state of the vibrational mode [Eq. (4)] has disentangled from that of the Stokes mode, so that expectation values of products of operators in their joint state factorize into products of expectation values; thus,

$$
g_{\mathrm{AS} \mid \mathrm{S}}^{(2)}(t>0)=\frac{\left\langle\hat{b}^{\dagger}(t) \hat{b}^{\dagger}(t) \hat{b}(t) \hat{b}(t)\right\rangle_{\mid \mathrm{S}}}{\left\langle\hat{b}^{\dagger}(t) \hat{b}(t)\right\rangle_{\mid \mathrm{S}}^{2}}=g_{b \mid \mathrm{S}}^{(2)} .
$$

That is, the conditional intensity correlation of the antiStokes field gives the intensity correlation of the vibrational mode. Immediately after the write pulse, i.e., at $t=0$, and in the limit of a small probability $p$ of exciting a vibrational Fock state, explicit evaluation of Eq. (6) on the state $|\Psi\rangle_{\mathrm{S}, b}$ of Eq. (3) yields

$$
g_{\mathrm{AS} \mid \mathrm{S}}^{(2)}(0) \approx \frac{4 P(2,2)}{P(1,1)}=4 p,
$$

where $P(n, n)=\left\langle n, n\left|\left(|\Psi\rangle_{\mathrm{S}, b}\langle\Psi|\right)\right| n, n\right\rangle=p^{n}$ is the probability of finding $n$ pairs of excitations in the vibrational mode and Stokes field upon a projective measurement on state Eq. (3) in the Fock state basis.

In our experiment we measure the number of events $N_{d_{1}, d_{2}, a_{\mathrm{S}}}$ where photons were detected simultaneously in modes $d_{1}, d_{2}$, and $a_{\mathrm{S}}$ (i.e., triple coincidence) and 
normalize it to the product of the number of events $N_{d_{i}, a_{S}}$ $(i=1,2)$ where photons are detected simultaneously in the Stokes mode and one of the anti-Stokes detectors (i.e., a twofold coincidence); we thus measure [23]

$$
\alpha \equiv \frac{N_{d_{1}, d_{2}, a_{\mathrm{s}}} N_{a_{\mathrm{S}}}}{N_{d_{1}, a_{\mathrm{S}}} N_{d_{2}, a_{\mathrm{S}}}} .
$$

It is important to note that, in general, $\alpha$ is not equivalent to $g_{\mathrm{AS} \mid \mathrm{S}}^{(2)}$ in Eq. (8). More precisely, if the detection efficiency of the herald mode $a_{\mathrm{S}}$ is $0<\eta \leq 1$ (which we model as a beam splitter with transmittance $\eta$ placed before the detector), we find (see Supplemental Material, Sec. IV [42])

$$
\alpha \approx(4-2 \eta) \frac{P(2,2)}{P(1,1)}=(4-2 \eta) p
$$

Thus, in the limit of low detection efficiency of Stokes photons (i.e., $\eta \ll 1$ ), we have $\alpha=g_{\mathrm{AS} \mid \mathrm{S}}^{(2)}$. In the experiment, $\eta \approx 10 \%$, so that $\alpha \approx g_{\mathrm{AS} \mid \mathrm{S}}^{(2)}$ (to within $5 \%$ ).

\section{EXPERIMENTAL REALIZATION}

\section{A. Setup and measurement procedure}

Our experimental setup is an upgraded version of that presented in Ref. [41]. Two synchronized laser pulse trains at 810 and $695 \mathrm{~nm}$ of duration $\Delta t \approx 100 \mathrm{fs}$ are produced by a Ti:sapphire oscillator (Tsunami, Spectra Physics, $80-\mathrm{MHz}$ repetition rate) and a synchronously pumped frequency-doubled optical parametric oscillator (OPO-X fs, APE Berlin), respectively. The write pulses are provided by the OPO, while the Ti:sapphire provides the read pulses, which are passed through a delay line before being overlapped with the OPO output on a dichroic mirror. The sample is a synthetic diamond crystal $(\sim 300 \mu \mathrm{m}$ thick, from LakeDiamond) cut along the (100) crystal plane and is probed in transmission using two microscope objectives (numerical aperture 0.8 and 0.9). The laser light is blocked using long-pass and short-pass tunable interference filters (Semrock), leaving only a spectral window of transmission for the Stokes signal from the write pulse (mode $a_{\mathrm{S}}$ ) and the anti-Stokes signal from the read pulse (mode $a_{\mathrm{AS}}$ ). The transmission is collected in a single mode fiber (for spatial mode filtering) and then the two signals are separated with a tunable long-pass filter used as a dichroic mirror. After an additional bandpass filter (see Supplemental Material, Sec. VI [42]) each signal is coupled into a multimode fiber; subsequently, the Stokes field is sent to a singlephoton counting module (SPCM, Excelitas), while the antiStokes field is split at a 50:50 fiber beam splitter and directed onto two SPCMs. The three SPCMs are then connected to a coincidence counter (PicoQuant TimeHarp 260).
We only record the coincidence events where a click in one of the anti-Stokes channels was preceded by a click in the Stokes channel. This allows us to find heralded coincidence events (within the same laser repetition), as well as to build a delay histogram using the Stokes channel as the start and either of the anti-Stokes channels as the stop. These start-stop histograms are used to compute the Stokes-anti-Stokes intensity cross-correlation as explained in Ref. [41]. Therefore, all relevant coincidences required to estimate $\alpha$ are available.

\section{B. Ambient thermal state}

We start by verifying that following the write pulse the Stokes field is well described by the state of Eq. (3): when marginalized over the state of the vibrational mode, the Stokes field is thermal. Indeed, we find in Fig. 2 (red bars) that the intensity correlation function of the Stokes field at zero time delay is $g_{\mathrm{S}}^{(2)}(0)=2$, as expected for a singlemode thermal state. Similarly, in the absence of the write operation, the anti-Stokes signal should reflect the thermal statistics of the vibrational mode. To check this, we measure the (unconditional) intensity correlation of the anti-Stokes mode, shown in Fig. 2 (blue bars). The value

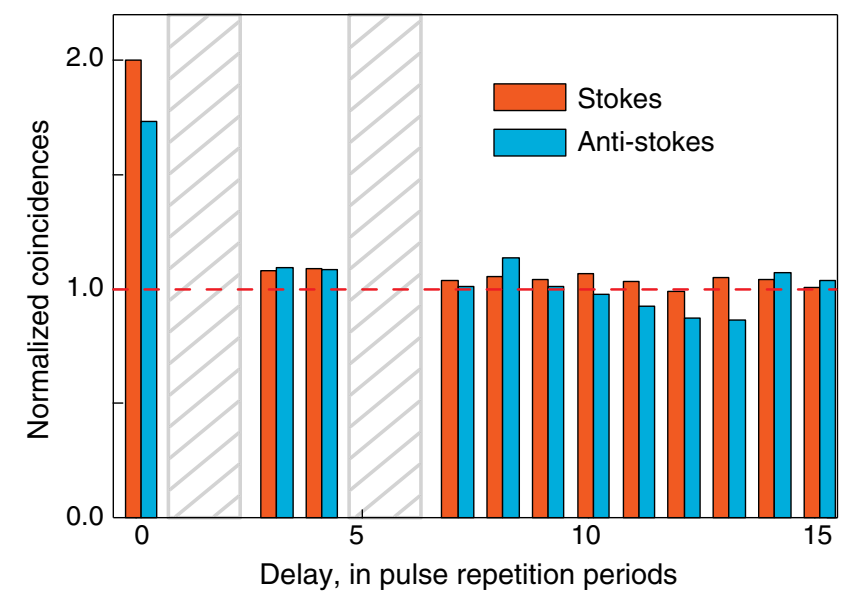

FIG. 2. Unconditional Stokes and anti-Stokes correlations. Twophoton coincidence histograms of the Stokes field $\hat{a}_{\mathrm{S}}$ (write pulse energy $60 \mathrm{pJ}$, acquisition time $10 \mathrm{~min}$ ) and anti-Stokes field $\hat{a}_{\mathrm{AS}}$ (read pulse energy $372 \mathrm{pJ}$, acquisition time $60 \mathrm{~min}$ ). For the antiStokes field the coincidences are recorded between the detectors measuring $\hat{d}_{1}$ and $\hat{d}_{2}$ as in Fig. 1(b), while the write pulse is blocked. For the measurement of the Stokes field, a beam splitter is added in the path of mode $\hat{a}_{\mathrm{S}}$. The start-stop delay (horizontal axis) is scaled in multiples of the repetition period $\approx 12.5 \mathrm{~ns}$. After normalizing by the average number of accidental coincidences (the peaks at nonzero start-stop), the value at zero time delay represents the intensity correlation of the Stokes and anti-Stokes fields; namely, $g_{\mathrm{S}}^{(2)}(0)=2.0 \pm 0.1$ and $g_{\mathrm{AS}}^{(2)}(0)=1.73 \pm 0.11$. The hatched regions in gray, omitted in the analysis, are affected by spurious coincidences due to cross talk between the two detectors, which arise when hot-carrier-induced light emission from one detector [47] is received by the other detector. 
of $g_{\mathrm{AS}}^{(2)}(0)=1.73 \pm 0.11$ is slightly lower than the expected value of 2 for a single mode thermal state, but higher than the value $1+(1 / N)$ for a thermal state of $N>1$ modes [46]. We attribute this discrepancy to noise in the antiStokes channel coming from degenerate four-wave mixing in the sample (which includes the second-order Stokesanti-Stokes process [37] discussed earlier). We thus confirm a single vibrational mode in an ambient thermal state, and that the result of the write operation is well described by the two-mode squeezed state of Eq. (3).

\section{Fock state preparation}

In order to prepare the vibrational mode in a Fock state, we send a write pulse and herald the success of this operation by detecting a Stokes photon. When a subsequent read pulse retrieves the vibrational state, and is subjected to intensity correlation measurements, we find that $\alpha(0) \approx$ $g_{\mathrm{AS} \mid \mathrm{S}}^{(2)}(0)<1$, as shown in Fig. 3 (main panel). Thus, the conditional anti-Stokes field exhibits sub-Poissonian statistics. But since we know that the anti-Stokes field is faithful to the vibrational state, and specifically that $g_{\mathrm{AS} \mid \mathrm{S}}^{(2)}=$ $g_{b \mid \mathrm{S}}^{(2)}$, we are able to conclude that the vibrational mode exhibits sub-Poissonian number statistics. From the value of $\alpha(0) \approx 0.06$ at the lowest powers of the write pulse and

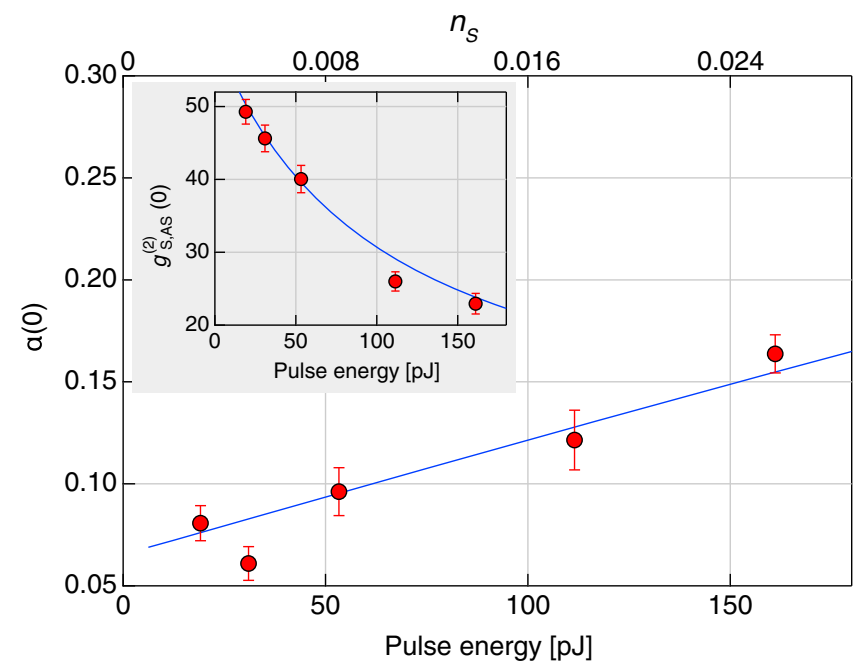

FIG. 3. Sub-Poissonian anti-Stokes statistics. Dependence of the heralded vibrational statistics on the write pulse energy and on the corresponding estimated probability of creating at least one Stokes photon per pulse, $n_{\mathrm{S}}=[p /(1-p)]$. The normalized Stokes-anti-Stokes correlations are shown as an inset. The write-read pulse delay is fixed at zero and the read pulse energy at $322 \mathrm{pJ}$. Statistical error bars are obtained from the square root of the total number of events. Blue lines are models (see Supplemental Material, Sec. V[42]), using the estimated detection efficiency $(10 \%)$ and a relative efficiency of the read process (relative to the Stokes emission cross section) of $30 \%$ as the only two adjustable parameters (common to both panels). the known detection efficiency of the Stokes field $\eta \approx 10 \%$, our theoretical model allows us to estimate the probability of having excited the Fock state $|1\rangle$ to be [Eq. (10)] $1-p \approx 98.5 \%$.

With increasing power of the write pulse, mixtures of states higher up in the Fock ladder are excited. As shown in Fig. 3, the sub-Poissonian character of $\alpha$ decreases with increasing pump power, as expected from the simple model $\alpha(t=0) \propto p=\tanh ^{2}\left(g_{\mathrm{S}}^{w} T_{w}\right) \approx\left(g_{\mathrm{S}}^{w} T_{w}\right)^{2} \propto n_{p}^{w}$, where $n_{p}^{w}$ is the number of photons per write pulse. In tandem, the Stokes-anti-Stokes correlation reduces as $1 / n_{p}^{w}$ (Fig. 3, inset). These trends are consistent with increasing probability of exciting two or more vibrational quanta $[46,48]$ (see Supplemental Material, Sec. V [42]).

\section{Fock state dynamics}

The decay of the excited vibrational Fock state can be probed by allowing it to evolve freely after the write pulse. In the experiment, we do this by employing a variable optical path length to impose a time delay $t$ between the write and read pulses. Figure 4 summarizes the observed time dependence of the excited Fock state. When the write and read pulses overlap $(t=0)$, we observe strong Stokesanti-Stokes number correlation $g_{\mathrm{S}, \mathrm{AS}}^{(2)}(0) \approx 30$, consistent with the generation of a Stokes-vibration two-mode squeezed state [Eq. (3)].

Simultaneously, $\alpha$, which reflects the intensity correlation of the conditioned vibrational state [Eq. (4)], indicates sub-Poissonian statistics of the vibrational mode, with $\alpha(t=0) \approx 0.11<1$. Conditioned on the detection of a Stokes photon, the vibrational mode is thus faithfully prepared in the Fock state $|1\rangle$.

Subsequent iterations of the experiment probe the vibrational state after a controlled time delay. Figure 4(a) shows the decay of the Stokes-anti-Stokes correlation. The initial value $g_{\mathrm{S}, \mathrm{AS}}^{(2)}(0)$ quantifies the degree to which the Stokes field and vibrational mode are correlated by the write operation. At later times $(t>0)$, i.e., after the Stokes field is detected, $g_{\mathrm{S}, \mathrm{AS}}^{(2)}(t)$ is proportional to $\left\langle\hat{b}^{\dagger}(t) \hat{b}(t)\right\rangle_{\mid \mathrm{S}}$ and the data in Fig. 4(a) can be used in conjunction with a model (shown in blue, consisting of the ideal prediction convolved with the known instrument response) to infer the decay rate $\tau_{m}=3.9 \pm 0.3 \mathrm{ps}$ (bounds for 95\% confidence). This value is consistent with the previously reported vibrational lifetime of 3.6 ps [25].

In parallel, as shown in Fig. 4(b), $\alpha(t)$ mirrors this evolution, starting at $\alpha(t<0)=1.9 \pm 0.6$ (thermal state), dropping to $\alpha(0)=0.11 \pm 0.01$ at zero delay (Fock state $|1\rangle)$, and then returning toward its equilibrium value as the prepared vibrational Fock state thermalizes with its environment. (The larger uncertainty in the data at long and at negative delays is due to the reduced rate of coincidences, because of the small thermal occupancy, $\bar{n} \approx 1.5 \times 10^{-3}$, of the vibrational mode.) This behavior is captured by a 

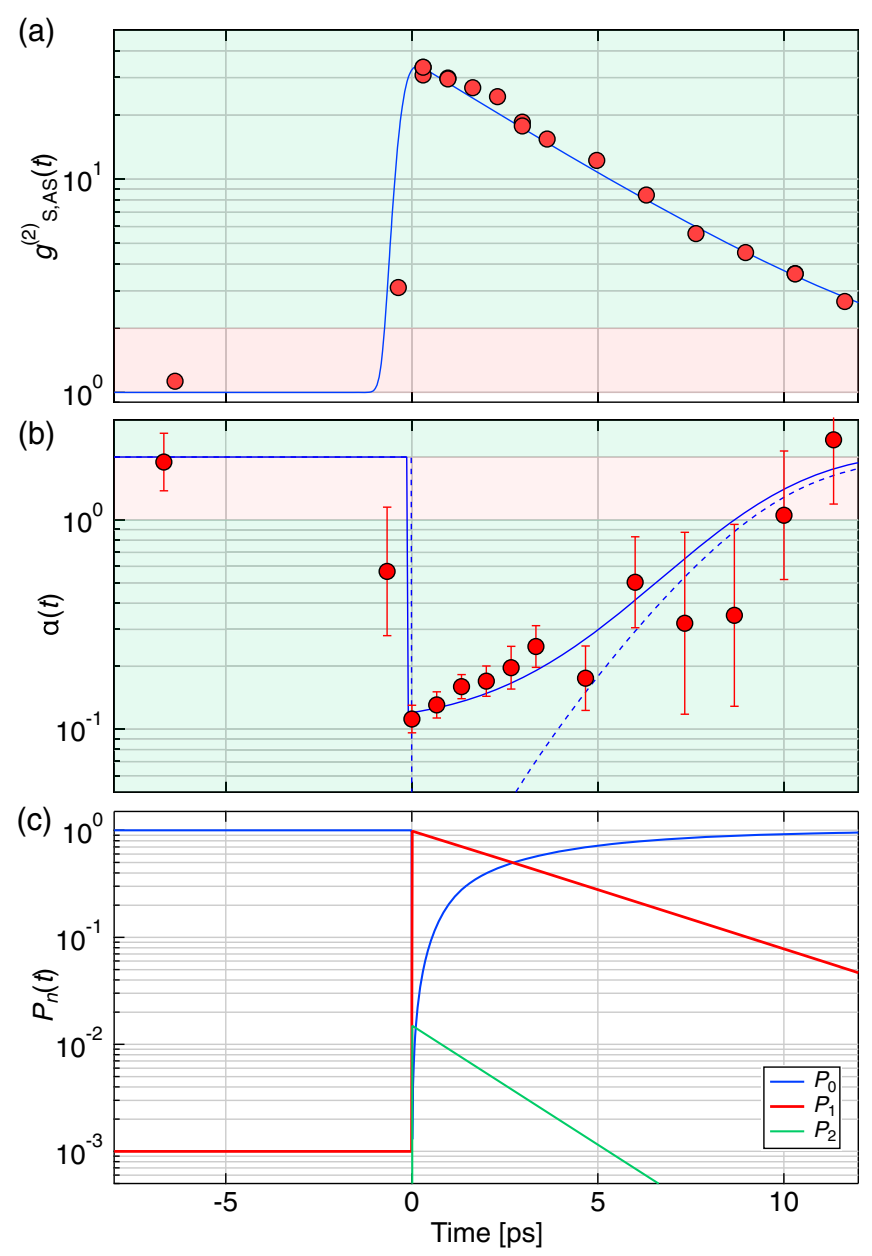

FIG. 4. Decay of vibrational Fock state. Measured Stokes-antiStokes correlations (a) and heralded vibrational mode intensity correlation (b) as a function of write-read delay. The measurements (full circles) are taken with a pulse energy of $62 \mathrm{pJ}$ in the write pulse and $409 \mathrm{pJ}$ in the read pulse, with an acquisition time of $60 \mathrm{~min}$ for all points except the one at $-6.3 \mathrm{ps}$, which was acquired over $8 \mathrm{~h}$. Statistical error bars are obtained using the method described in Ref. [17]. Full circles are measured data and blue lines are from the model; see main text and Eq. (11). The prediction from the model without the added noise on the antiStokes detectors is shown with a dashed line in (b). Green bands in panels (a) and (b) show the region where a nonclassical model is required to explain the observations; red bands indicate where a classical model suffices. (c) Fock state distribution of the conditional vibrational mode inferred from the data via our model.

simple model [shown as a blue line in Fig. 4(b)], based on the fact that $\alpha(t)$ is the intensity correlation of the vibrational mode [Eq. (7)]—which can be calculated using an open quantum system model for the vibrational modetogether with a contribution from background noise in the anti-Stokes field (see Supplemental Material, Sec. II.B [42]),

$$
\alpha(t) \approx \frac{2}{P_{1}(0)}\left[1-\frac{1}{\left[1+\bar{n}\left(e^{t / \tau_{m}}-1\right)\right]^{2}}\right]+\alpha_{0},
$$

where $P_{1}(0) \approx 0.985$ is the probability of having created the vibrational Fock state $|1\rangle$ conditioned upon the detection of a Stokes photon, and $\alpha_{0}=2 \times 0.04$ corresponds to twice the anti-Stokes noise-to-signal ratio conditioned on Stokes detection (i.e., $\alpha_{0} / 2 \approx 1 / g_{\mathrm{S}, \mathrm{AS}}^{(2)}(0)$; see Supplemental Material, Sec. III [42]). The decay of sub-Poissonian statistics of the vibrational mode is consistent with the decay of the Fock state $|1\rangle$ to the ground state $|0\rangle$.

The measured decay of the conditional intensity correlation, in conjunction with a decoherence model, can be used to extract the number distribution $P_{n}(t)$ of the conditional vibrational state (see Supplemental Material, Sec. II.A [42]) - the probability to find the vibrational mode in the Fock state $|n\rangle$ at time $t$ after a click on the Stokes detector. This projection is plotted in Fig. 4(c). Noteworthy is the high purity of the conditional vibrational state with respect to the Fock state $|n=1\rangle$, since its normalized second-order correlation is $\left[\left(2 P_{2}\right) /\left(P_{1}^{2}\right)\right]$ $\approx 0.02$. This is much lower than the measured parameter $\alpha \approx 0.1$ (because the background noise impinging on the anti-Stokes detectors affects $\alpha$ ) and highlights the potential of the technique to produce high-purity single phonons.

\section{CONCLUSION}

We have demonstrated for the first time that a highfrequency Raman-active vibrational mode can be prepared in its $n=1$ Fock state at room temperature. Heralded intensity correlation measurements confirm the sub-Poissonian statistics of the conditional vibrational state. We further probed the decay of the vibrational Fock state, akin to similar measurements on microwave photons [49,50].

This research opens a door to the study of quantum effects in the vibrational dynamics of Raman-active modes in immobilized molecules [51], liquids, gases [52-54], and solid-state systems. Vibrational states in Raman-active solidstate systems at room temperature may even be viable candidates for quantum technology if the coherence time and readout efficiency can be improved. Coherence of the longitudinal optical phonon modes of diamond is known to be limited by decay through the so-called Klemens channel [55-57]. Proposals to close this pathway include the creation of a phononic band gap at the atomic scale by growing ${ }^{12} \mathrm{C}-{ }^{13} \mathrm{C}$ super lattices [25]. Readout efficiency and heralding rate could be improved by coupling the Raman-active system to small-mode volume optical or plasmonic cavities [58-60], or by employing resonant Raman scattering [6,61]. Molecular systems are particularly promising for extending the vibrational lifetime [62]. With these improvements, vibrational modes may be used as a source of highpurity on-demand (anti-Stokes) photons, or as a buffer memory to produce heralded single photons with an arbitrary choice of the herald and signal wavelengths and/or bandwidths, or even heralded frequency conversion at the singlephoton level. 


\section{ACKNOWLEDGMENTS}

Funding for this research was provided by the Swiss National Science Foundation (Project No. PP00P2-170684). K. S. acknowledges support from the Swiss National Science Foundation (Project No. 200021-162357). V. S. is supported by a Swiss National Science Foundation fellowship (Project No. P2ELP2-178231). This research has received funding from the European Research Council (ERC) under the European Unions Horizon 2020 research and innovation programme (Grant Agreement No. 820196). C. G. thanks Philippe Grangier for valuable discussions.

[1] A. H. Compton, A Quantum Theory of the Scattering of X-Rays by Light Elements, Phys. Rev. 21, 483 (1923).

[2] C. V. Raman and K. S. Krishnan, A New Type of Secondary Radiation, Nature (London) 121, 501 (1928).

[3] D. von der Linde, J. Kuhl, and H. Klingenberg, Raman Scattering from Nonequilibrium LO Phonons with Picosecond Resolution, Phys. Rev. Lett. 44, 1505 (1980).

[4] J. A. Kash, J. C. Tsang, and J. M. Hvam, Subpicosecond Time-Resolved Raman Spectroscopy of LO Phonons in GaAs, Phys. Rev. Lett. 54, 2151 (1985).

[5] D. Song, F. Wang, G. Dukovic, M. Zheng, E. D. Semke, L. E. Brus, and T. F. Heinz, Direct Measurement of the Lifetime of Optical Phonons in Single-Walled Carbon Nanotubes, Phys. Rev. Lett. 100, 225503 (2008).

[6] T. Jiang, H. Hong, C. Liu, W.-T. Liu, K. Liu, and S. Wu, Probing Phonon Dynamics in Individual Single-Walled Carbon Nanotubes, Nano Lett. 18, 2590 (2018).

[7] S. Mukamel, Principles of Nonlinear Optical Spectroscopy (Oxford University Press, New York, 1995), Vol. 29.

[8] F. C. Waldermann, B. J. Sussman, J. Nunn, V. O. Lorenz, K. C. Lee, K. Surmacz, K. H. Lee, D. Jaksch, I. A. Walmsley, P. Spizziri, P. Olivero, and S. Prawer, Measuring Phonon Dephasing with Ultrafast Pulses Using Raman Spectral Interference, Phys. Rev. B 78, 155201 (2008).

[9] K. C. Lee, B. J. Sussman, J. Nunn, V. O. Lorenz, K. Reim, D. Jaksch, I. A. Walmsley, P. Spizzirri, and S. Prawer, Comparing Phonon Dephasing Lifetimes in Diamond Using Transient Coherent Ultrafast Phonon Spectroscopy, Diam. Relat. Mater. 19, 1289 (2010).

[10] G. A. Garrett, A. G. Rojo, A. K. Sood, J. F. Whitaker, and R. Merlin, Vacuum Squeezing of Solids: Macroscopic Quantum States Driven by Light Pulses, Science 275, 1638 (1997).

[11] K. C. Lee, M. R. Sprague, B. J. Sussman, J. Nunn, N. K. Langford, X.-M. Jin, T. Champion, P. Michelberger, K. F. Reim, D. England, D. Jaksch, and I. A. Walmsley, Entangling Macroscopic Diamonds at Room Temperature, Science 334, 1253 (2011).

[12] M. Aspelmeyer, T. J. Kippenberg, and F. Marquardt, Cavity Optomechanics, Rev. Mod. Phys. 86, 1391 (2014).

[13] T. P. Purdy, K. E. Grutter, K. Srinivasan, and J. M. Taylor, Quantum Correlations from a Room-Temperature Optomechanical Cavity, Science 356, 1265 (2017).

[14] V. Sudhir, R. Schilling, S. A. Fedorov, H. Schütz, D. J. Wilson, and T. J. Kippenberg, Quantum Correlations of
Light from a Room-Temperature Mechanical Oscillator, Phys. Rev. X 7, 031055 (2017).

[15] J. Cripe, N. Aggarwal, R. Lanza, A. Libson, R. Singh, P. Heu, D. Follman, G. D. Cole, N. Mavalvala, and T. Corbitt, Measurement of Quantum Back Action in the Audio Band at Room Temperature, Nature (London) 568, 364 (2019).

[16] E. E. Wollman, C. U. Lei, A. J. Weinstein, J. Suh, A. Kronwald, F. Marquardt, A. A. Clerk, and K. C. Schwab, Quantum Squeezing of Motion in a Mechanical Resonator, Science 349, 952 (2015).

[17] S. Hong, R. Riedinger, I. Marinković, A. Wallucks, S. G. Hofer, R. A. Norte, M. Aspelmeyer, and S. Gröblacher, Hanbury Brown and Twiss Interferometry of Single Phonons from an Optomechanical Resonator, Science 358, 203 (2017).

[18] R. Riedinger, A. Wallucks, I. Marinković, C. Löschnauer, M. Aspelmeyer, S. Hong, and S. Gröblacher, Remote Quantum Entanglement between Two Micromechanical Oscillators, Nature (London) 556, 473 (2018).

[19] C. F. Ockeloen-Korppi, E. Damskägg, J.-M. Pirkkalainen, M. Asjad, A. A. Clerk, F. Massel, M. J. Woolley, and M. A. Sillanpää, Stabilized Entanglement of Massive Mechanical Oscillators, Nature (London) 556, 478 (2018).

[20] Y. Chu, P. Kharel, T. Yoon, L. Frunzio, P. T. Rakich, and R. J. Schoelkopf, Creation and Control of Multi-Phonon Fock States in a Bulk Acoustic Wave Resonator, Nature (London) 563, 666 (2018).

[21] L. M. Duan, M. D. Lukin, J. I. Cirac, and P. Zoller, LongDistance Quantum Communication with Atomic Ensembles and Linear Optics, Nature (London) 414, 413 (2001).

[22] C. Galland, N. Sangouard, N. Piro, N. Gisin, and T. J. Kippenberg, Heralded Single-Phonon Preparation, Storage, and Readout in Cavity Optomechanics, Phys. Rev. Lett. 112, 143602 (2014).

[23] P. Grangier, G. Roger, and A. Aspect, Experimental Evidence for a Photon Anticorrelation Effect on a Beam Splitter: A New Light on Single-Photon Interferences, Europhys. Lett. 1, 173 (1986).

[24] L. Mandel and E. Wolf, Optical Coherence and Quantum Optics (Cambridge University Press, Cambridge, England, 1995).

[25] K. C. Lee, B. J. Sussman, M. R. Sprague, P. Michelberger, K. F. Reim, J. Nunn, N. K. Langford, P. J. Bustard, D. Jaksch, and I. A. Walmsley, Macroscopic Non-Classical States and Terahertz Quantum Processing in RoomTemperature Diamond, Nat. Photonics 6, 41 (2012).

[26] D. G. England, P. J. Bustard, J. Nunn, R. Lausten, and B. J. Sussman, From Photons to Phonons and Back: A $\mathrm{THz}$ Optical Memory in Diamond, Phys. Rev. Lett. 111, 243601 (2013).

[27] D. G. England, K. A. G. Fisher, J.-P. W. MacLean, P. J. Bustard, R. Lausten, K. J. Resch, and B. J. Sussman, Storage and Retrieval of THz-Bandwidth Single Photons Using a Room-Temperature Diamond Quantum Memory, Phys. Rev. Lett. 114, 053602 (2015).

[28] P.-Y. Hou, Y.-Y. Huang, X.-X. Yuan, X.-Y. Chang, C. Zu, L. He, and L.-M. Duan, Quantum Teleportation from Light Beams to Vibrational States of a Macroscopic Diamond, Nat. Commun. 7, 11736 (2016). 
[29] K. A. G. Fisher, D. G. England, J.-P. W. MacLean, P. J. Bustard, K. J. Resch, and B. J. Sussman, Frequency and Bandwidth Conversion of Single Photons in a RoomTemperature Diamond Quantum Memory, Nat. Commun. 7, 11200 (2016).

[30] K. A. G. Fisher, D. G. England, J.-P. W. MacLean, P. J. Bustard, K. Heshami, K. J. Resch, and B. J. Sussman, Storage of Polarization-Entangled THz-Bandwidth Photons in a Diamond Quantum Memory, Phys. Rev. A 96, 012324 (2017).

[31] H. Flayac and V. Savona, Heralded Preparation and Readout of Entangled Phonons in a Photonic Crystal Cavity, Phys. Rev. Lett. 113, 143603 (2014).

[32] V. C. Vivoli, T. Barnea, C. Galland, and N. Sangouard, Proposal for an Optomechanical Bell Test, Phys. Rev. Lett. 116, 070405 (2016).

[33] I. Marinković, A. Wallucks, R. Riedinger, S. Hong, M. Aspelmeyer, and S. Gröblacher, Optomechanical Bell Test, Phys. Rev. Lett. 121, 220404 (2018).

[34] T. von Foerester and R. J. Glauber, Quantum Theory of Light Propagation in Amplifying Media, Phys. Rev. A 3, 1484 (1971).

[35] N. Bloembergen, Nonlinear Optics (W. A. Benjamin, New York, 1965).

[36] M. Kasperczyk, A. Jorio, E. Neu, P. Maletinsky, and L. Novotny, Stokes-Anti-Stokes Correlations in Diamond, Opt. Lett. 40, 2393 (2015).

[37] C. A. Parra-Murillo, M. F. Santos, C. H. Monken, and A. Jorio, Stokes-Anti-Stokes Correlation in the Inelastic Scattering of Light by Matter and Generalization of the Bose-Einstein Population Function, Phys. Rev. B 93, 125141 (2016).

[38] M. K. Schmidt, R. Esteban, A. González-Tudela, G. Giedke, and J. Aizpurua, Quantum Mechanical Description of Raman Scattering from Molecules in Plasmonic Cavities, ACS Nano 10, 6291 (2016).

[39] M. Kasperczyk, F. S. de Aguiar Júnior, C. Rabelo, A. Saraiva, M. F. Santos, L. Novotny, and A. Jorio, Temporal Quantum Correlations in Inelastic Light Scattering from Water, Phys. Rev. Lett. 117, 243603 (2016).

[40] A. Saraiva, F. S. de Aguiar Júnior, R. de Melo e Souza, A. P. Pena, C. H. Monken, M. F. Santos, B. Koiller, and A. Jorio, Photonic Counterparts of Cooper Pairs, Phys. Rev. Lett. 119, 193603 (2017).

[41] M. D. Anderson, S. Tarrago Velez, K. Seibold, H. Flayac, V. Savona, N. Sangouard, and C. Galland, Two-Color PumpProbe Measurement of Photonic Quantum Correlations Mediated by a Single Phonon, Phys. Rev. Lett. 120, 233601 (2018).

[42] See Supplemental Material at http://link.aps.org/ supplemental/10.1103/PhysRevX.9.041007 for a theoretical model of the dynamics of the phonon Fock state, and further details regarding the experiment and its systematics.

[43] A. J. Weinstein, C. U. Lei, E. E. Wollman, J. Suh, A. Metelmann, A. A. Clerk, and K. C. Schwab, Observation and Interpretation of Motional Sideband Asymmetry in a Quantum Electromechanical Device, Phys. Rev. X 4, 041003 (2014).
[44] E Knill, R Laflamme, and G. J. Milburn, A Scheme for Efficient Quantum Computation with Linear Optics, Nature (London) 409, 46 (2001).

[45] R. J. Glauber, The Quantum Theory of Optical Coherence, Phys. Rev. 130, 2529 (1963).

[46] P. Sekatski, N. Sangouard, F. Bussieres, C. Clausen, N. Gisin, and H. Zbinden, Detector Imperfections in Photon-Pair Source Characterization, J. Phys. B 45, 124016 (2012).

[47] A. L. Lacaita, F. Zappa, S. Bigliardi, and M. Manfredi, On the Bremsstrahlung Origin of Hot-Carrier-Induced Photons in Silicon Devices, IEEE Trans. Electron Devices 40, 577 (1993).

[48] S. Friberg, C. K. Hong, and L. Mandel, Intensity Dependence of the Normalized Intensity Correlation Function in Parametric Down-Conversion, Opt. Commun. 54, 311 (1985).

[49] H. Wang, M. Hofheinz, M. Ansmann, R. C. Bialczak, E. Lucero, M. Neeley, A. D. O'Connell, D. Sank, J. Wenner, A. N. Cleland, and John M. Martinis, Measurement of the Decay of Fock States in a Superconducting Quantum Circuit, Phys. Rev. Lett. 101, 240401 (2008).

[50] M. Brune, J. Bernu, C. Guerlin, S. Deléglise, C. Sayrin, S. Gleyzes, S. Kuhr, I. Dotsenko, J. M. Raimond, and S. Haroche, Process Tomography of Field Damping and Measurement of Fock State Lifetimes by Quantum Nondemolition Photon Counting in a Cavity, Phys. Rev. Lett. 101, 240402 (2008).

[51] S. Yampolsky, D. A. Fishman, S. Dey, E. Hulkko, M. Banik, E. O. Potma, and V. A. Apkarian, Seeing a Single Molecule Vibrate through Time-Resolved Coherent Anti-Stokes Raman Scattering, Nat. Photonics 8, 650 (2014).

[52] P. J. Bustard, R. Lausten, D. G. England, and B. J. Sussman, Toward Quantum Processing in Molecules: A THz-Bandwidth Coherent Memory for Light, Phys. Rev. Lett. 111, 083901 (2013).

[53] P. J. Bustard, J. Erskine, D. G. England, J. Nunn, P. Hockett, R. Lausten, M. Spanner, and B. J. Sussman, Nonclassical Correlations between Terahertz-Bandwidth Photons Mediated by Rotational Quanta in Hydrogen Molecules, Opt. Lett. 40, 922 (2015).

[54] P. J. Bustard, D. G. England, K. Heshami, C. Kupchak, and B. J. Sussman, Reducing Noise in a Raman Quantum Memory, Opt. Lett. 41, 5055 (2016).

[55] P. G. Klemens, Anharmonic Decay of Optical Phonons, Phys. Rev. 148, 845 (1966).

[56] A. Debernardi, S. Baroni, and E. Molinari, Anharmonic Phonon Lifetimes in Semiconductors from DensityFunctional Perturbation Theory, Phys. Rev. Lett. 75, 1819 (1995).

[57] M. S. Liu, L. A. Bursill, S. Prawer, and R. Beserman, Temperature Dependence of the First-Order Raman Phonon Line of Diamond, Phys. Rev. B 61, 3391 (2000).

[58] P. Roelli, C. Galland, N. Piro, and T. J. Kippenberg, Molecular Cavity Optomechanics as a Theory of Plasmon-Enhanced Raman Scattering, Nat. Nanotechnoly 11, 164 (2016).

[59] F. Benz, M. K. Schmidt, A. Dreismann, R. Chikkaraddy, Y. Zhang, A. Demetriadou, C. Carnegie, H. Ohadi, B. de Nijs, R. Esteban, J. Aizpurua, and J. J. Baumberg, 
Single-Molecule Optomechanics in "Picocavities," Science 354, 726 (2016).

[60] A. Lombardi, M. K. Schmidt, L. Weller, W. M. Deacon, F. Benz, B. de Nijs, J. Aizpurua, and J. J. Baumberg, Pulsed Molecular Optomechanics in Plasmonic Nanocavities: From Nonlinear Vibrational Instabilities to Bond-Breaking, Phys. Rev. X 8, 011016 (2018).
[61] A. Jorio, M. Kasperczyk, N. Clark, E. Neu, P. Maletinsky, A. Vijayaraghavan, and L. Novotny, Optical-Phonon Resonances with Saddle-Point Excitons in Twisted-Bilayer Graphene, Nano Lett. 14, 5687 (2014).

[62] L. Chen, J. A. Lau, D. Schwarzer, J. Meyer, V. B. Verma, and A. M. Wodtke, The Sommerfeld Ground-Wave Limit for a Molecule Adsorbed at a Surface, Science 363, 158 (2019). 